Research Article

\title{
Chemical Control for Host-Parasitoid Model within the Parasitism Season and Its Complex Dynamics
}

\author{
Tao Wang ${ }^{1,2}$ and Youtang Zhang \\ ${ }^{1}$ School of Management, Wuhan University of Technology, Wuhan, Hubei 430070, China \\ ${ }^{2}$ School of Economics and Management, Hubei University for Nationalities, Enshi, Hubei 445000, China \\ Correspondence should be addressed to Tao Wang; wangtaoyuan261@163.com
}

Received 26 November 2015; Accepted 28 February 2016

Academic Editor: Rigoberto Medina

Copyright (c) 2016 T. Wang and Y. Zhang. This is an open access article distributed under the Creative Commons Attribution License, which permits unrestricted use, distribution, and reproduction in any medium, provided the original work is properly cited.

In the present paper, we develop a host-parasitoid model with Holling type II functional response function and chemical control, which can be applied at any time of each parasitism season or pest generation, and focus on addressing the importance of the timing of application pesticide during the parasitism season or pest generation in successful pest control. Firstly, the existence and stability of both the host and parasitoid populations extinction equilibrium and parasitoid-free equilibrium have been investigated. Secondly, the effects of key parameters on the threshold conditions have been discussed in more detail, which shows the importance of pesticide application times on the pest control. Thirdly, the complex dynamics including multiple attractors coexistence, chaotic behavior, and initial sensitivity have been studied by using numerical bifurcation analyses. Finally, the uncertainty and sensitivity of all the parameters on the solutions of both the host and parasitoid populations are investigated, which can help us to determine the key parameters in designing the pest control strategy. The present research can help us to further understand the importance of timings of pesticide application in the pest control and to improve the classical chemical control and to make management decisions.

\section{Introduction}

Integrated pest management (IPM) is the selection and application of pest control actions that can ensure favourable economic and ecological consequences [1-3], which has been successfully applied to agricultural pest management situations. IPM employs a variety of tactics including cultural controls, biological controls, and chemical controls or pesticides, while biological pest control is one of the most common measures applied in IPM through the control and management of natural predators and parasites. For example, mosquitoes are often controlled by putting Bacillus thuringiensis (Bt) ssp. israelensis, a bacterium that infects and kills mosquito larvae, in local water sources. The aim of biological pest control is to eliminate a pest which can minimize harm to the ecological balance of the environment in its present form.

Pesticide application or chemical control is another important component of IPM measures, which refers to the practical way in which pesticides including herbicides, fungicides, and insecticides are sprayed to the pest population. Public concern about the application of pesticides has highlighted the need to make this process as efficiently as possible which could minimize their release into the environment and human exposure [1-3]. Although pesticide and insecticide applications can cause a number of problems, in most cropping systems they are still the principle means of controlling pests once the economic threshold that defines the lowest population density at which the control actions should be applied has been reached. In practice, pesticides can be relatively cheap, are easy to apply and fast-acting, and in most instances can be relied on to control the pests [1-3].

Undoubtedly, mathematical model is one of key tools to help us to understand those factors in the IPM strategies and the pest control. Recently, the continuous predator-prey models concerning IPM strategy have been developed and investigated [1, 4-10]. In particular, several factors including pest natural enemy ratios, starting densities, timings of natural enemy releases, dosages and timings of insecticide 
applications, and instantaneous killing rates of pesticides on both pests and natural enemies have been addressed by Tang et al. [10]. Moreover, the importance of timings of pesticide sprays and natural enemy releases has been studied through the stability threshold condition for a pest eradication periodic solution.

However, the discrete or nonoverlapping generation is a common feature among the host and parasitoid populations [11-15]. If so the use of continuous-time models to describe the interaction between the pest population and its natural enemy population becomes questionable. Those show that the discrete host-parasitoid models are much more realistic when the populations have discrete and synchronized generations [16-21]. The complex dynamics of the host-parasitoid model with Holling II functional response function have been investigated by Tang and Chen [19]. The classical NicholsonBailey model for a two species' host-parasitoid system with discrete generations and IPM strategies has been studied by Tang et al. [7]. Note that the pulse IPM control strategies assumed to be applied at the end of each periodic number of generations and some important issues concerning IPM have been addressed.

According to the facts and main results of $[7,10]$, we know that the timings of pesticide application and releasing natural enemies play a key role in successful pest control. Note that there are several different pesticide spraying methods based on the pest growth generations and the parasitism season: (1) the pesticides are applied at the beginning of each generation; (2) the pesticides are sprayed at the end of each generation; and (3) the pesticides can be applied at any time point within the pest growth generation and the parasitism season. Therefore, the questions are whether there exists an optimal pesticide application time at which the number of the pest populations can be minimized, even eradicated, and how the pesticide application time and efficiency affect the successful pest control and complex outbreak patterns.

To address those questions, it follows from the basic modelling methods proposed in literatures [22-24] that we assume that the chemical control tactic is applied instantaneously within the generation at any time. The main purpose is to extend the Holling II host-parasitoid model with a fraction of survival rate of parasitoid from one generation to next $[25,26]$ and then investigate the effects of timings of pesticide application and killing rate on this model. In particular, the existence and stability of both the host and parasitoid populations extinction equilibrium and parasitoid-free equilibrium have been investigated. The interesting results indicate that the different pesticide application times could result in significantly different size of the host population and consequently influence the pest control. Moreover, the effects of all important parameters including timings of pesticide application on the threshold conditions have been discussed in more detail. Further, the complex dynamics including multiple attractors coexistence, quasiperiodic windows, chaotic behavior, and initial sensitivity have been studied by using numerical bifurcation analyses. Finally, the uncertainty and sensitivity of all the parameters on the solutions of both the host and parasitoid populations have been investigated, which can help us to determine the key parameters in designing the successful pest control strategy. The present research can help us to further understand the importance of pesticide application times in the pest control and to improve the classical chemical control and to make management decisions.

\section{The Host-Parasitoid Model with Chemical Control}

2.1. Host Growth Model with Chemical Control. The based discrete map for host or pest population can be described as follows:

$$
H_{n+1}=f\left(H_{n}\right)=H_{n} F\left(H_{n}\right)
$$

where $H_{n} \in[0, \infty)$ is the pest population size at generation $n \in \mathcal{N}$ and $f, F:[0, \infty) \rightarrow R$ are, respectively, the population production and the per capita production. A common example is the Rocker model $f(H)=H \exp (r(1-$ $H / K)$ ) with $r>0$ and $K>0$.

It follows from the basic modelling methods proposed in literatures [22-24] that we assume that the chemical control tactic is applied instantaneously within the generation $[n, n+$ $1]$; that is, there exists a positive constant $\theta$ with $0 \leq \theta \leq 1$ such that the pesticide is sprayed at time point $n+\theta$. Further, we assume that a proportional number (denoted by $q$ ) of the pest populations has been killed, and thus after $n+\theta$, the growth of pest population and its production depends on $(1-q) H_{n}$. Therefore, model (1) with chemical control can be formulated as

$$
H_{n+1}=(1-q) H_{n}\left[\theta F\left(H_{n}\right)+(1-\theta) F\left((1-q) H_{n}\right)\right] .
$$

According to $f\left(H_{n}\right)=H_{n} F\left(H_{n}\right)$, we have

$$
H_{n+1}=\theta(1-q) f\left(H_{n}\right)+(1-\theta) f\left((1-q) H_{n}\right) \text {. }
$$

2.2. Host-Parasitoid Model with the Effect of Overwintering Parasitoid and Chemical Control. Involving the parasitoid population into (1), the host-parasitoid system with discrete generations can be written $[16,17]$ as the generalized model

$$
\begin{aligned}
& H_{n+1} \\
& \quad=(1-q) H_{n}\left[\theta f\left(H_{n}\right)+(1-\theta) f\left((1-q) H_{n}\right)\right] \\
& \quad \cdot g\left((\theta+(1-\theta)(1-q)) H_{n}, P_{n}\right), \\
& \quad P_{n+1} \\
& \quad \gamma(1-q) H_{n}\left[1-g\left((\theta+(1-\theta)(1-q)) H_{n}, P_{n}\right)\right] \\
& \quad+\delta P_{n},
\end{aligned}
$$

where $H_{n}$ and $P_{n}$ represent the host and parasitoid population abundance in generation $n$, respectively, $f(H)$ denotes the per capita net rate of the increase of the host population in the absence of parasitoid population, and $g(H, P)$ is the proportion of host individuals that escape attack by the parasitoid. 
Note that $P$ is generally interpreted as the abundance of adult parasitoid females and $N$ as the abundance of host adults and thus $\gamma$ represents the number of parasitoid eggs laid per host, the survival of the parasitoid in the attacked hosts, and the sex ratio of the emerging parasitoid adults, where $\delta \epsilon$ $[0,1)$ denotes the density-independent survival of parasitoid propagules at generation $n$. A fraction of pathogen can survive from one generation to the next. This discrete-generation framework characterizes a perfectly synchronized parasitoid interacting with a host that has distinct generations, which is frequent in host-parasitoid systems in temperate regions of the world and even some from more tropical regions when parasitism causes generation cycles within the overlapping generations of some multivoltine hosts $[16,17]$.

In particular, if $\theta=0$ then model (4) can be changed as the following impulsive difference equation:

$$
\begin{aligned}
H_{n+1} & =H_{n} f\left(H_{n}\right) \cdot g\left(H_{n}, P_{n}\right) \\
P_{n+1} & =\gamma H_{n}\left[1-g\left(H_{n}, P_{n}\right)\right]+\delta P_{n}, \\
& n=0,1,2, \ldots, \\
H_{n^{+}} & =(1-q) H_{n}, \\
P_{n^{+}} & =P_{n},
\end{aligned}
$$

$$
n=0,1,2, \ldots,
$$

which indicates that the chemical control tactics have been applied at the beginning of each generation or parasitism season. For more details of impulsive difference equations, please see [7].

If $\theta=1$ then model (4) can be changed as the following impulsive difference equation:

$$
\begin{aligned}
H_{n+1} & =H_{n} f\left(H_{n}\right) \cdot g\left(H_{n}, P_{n}\right) \\
P_{n+1} & =\gamma H_{n}\left[1-g\left(H_{n}, P_{n}\right)\right]+\delta P_{n}, \\
& n=0,1,2, \ldots, \\
H_{(n+1)^{+}} & =(1-q) H_{n+1}, \\
P_{(n+1)^{+}} & =P_{n+1},
\end{aligned}
$$

which shows that the chemical control tactics have been applied at the ending of each generation or parasitism season.

Holling (1959) was the first ecologist to investigate the interaction between host and parasitoid populations and discussed the functional relationship in depth through the experiments of the shrews and deer mice feeding on sawfly cocoons. Holling (1959) presents three different functional response classes, for which the following are nominal forms: type I (linear then constant), type II (decelerating rise to an upper asymptote), and type III (sigmoidal) $[16,17,25,26]$. In the present work, we assume that the pest population follows the Ricker model in the absence of parasitoid population and the Holling II functional response function for parasitism; that is, we have

$$
\begin{gathered}
f\left(H_{n}\right)=\exp \left(r\left(1-\frac{H_{n}}{K}\right)\right), \\
g\left(H_{n}, P_{n}\right)=\exp \left(-\frac{\alpha T P_{n}}{1+\alpha T_{h} H_{n}}\right) .
\end{gathered}
$$

Therefore, based on the above special choices we will focus on the existence and stability of the boundary equilibrium which concerns the outbreak of the host population and then address how the timing of spraying pesticides affects the values of this boundary equilibrium. Moreover, the completely numerical bifurcation analyses and sensitivity analyses have been carried out to show the effects of interesting parameters including instant killing rate $q$ and pesticide spraying time point $\theta$ on the successful pest control and outbreak.

\section{Existence and Stability of Boundary Equilibria}

It is easy to see that model (4) has a zero equilibrium $(0,0)$ and its stability can be determined by the following Jacobian matrix:

$$
\begin{aligned}
& J_{(0,0)} \\
& \quad=\left(\begin{array}{cc}
(1-q)(\theta f(0,0)+(1-\theta) f(0,0)) g(0,0) & 0 \\
(1-q)(1-g(0,0)) & \delta
\end{array}\right) .
\end{aligned}
$$

It follows from (7) that the stability of $(0,0)$ can be determined by the eigenvalues of following Jacobian matrix:

$$
J_{(0,0)}=\left(\begin{array}{cc}
(1-q)\left(\theta e^{r}+(1-\theta) e^{r}\right) & 0 \\
0 & \delta
\end{array}\right) ;
$$

that is, $(0,0)$ is stable when the eigenvalues of the above matrix are less than one in magnitude. According to $0 \leq \delta<1$ we can see that the stability of $(0,0)$ is determined by the value of $(1-q) e^{r}$. This indicates that if $(1-q) e^{r}<1$, then $(0,0)$ is stable. Moreover, we note that the stability of $(0,0)$ only depends on the intrinsic growth of the host population and the instant killing rate $q$, which means that both the host and parasitoid populations could die out if the pesticide is effective enough no matter when we spray the pesticides.

Although the timing of pesticide applications (i.e., $\theta$ ) does not affect the stability of $(0,0)$, from Figure 1 we note that it can significantly influence the behavior of solutions of model (4). For example, letting the parameter $\theta$ vary and fixing for all others as those in Figure 1 at which $(1-q) e^{r}<1$ holds true and further letting all solutions start from the same initial value, we can see that the host population tends to zero more quickly for small values of $\theta$. This confirms that we should apply the pesticides at the ending of parasitism season or population generation, which could be best for the host control in practice. 


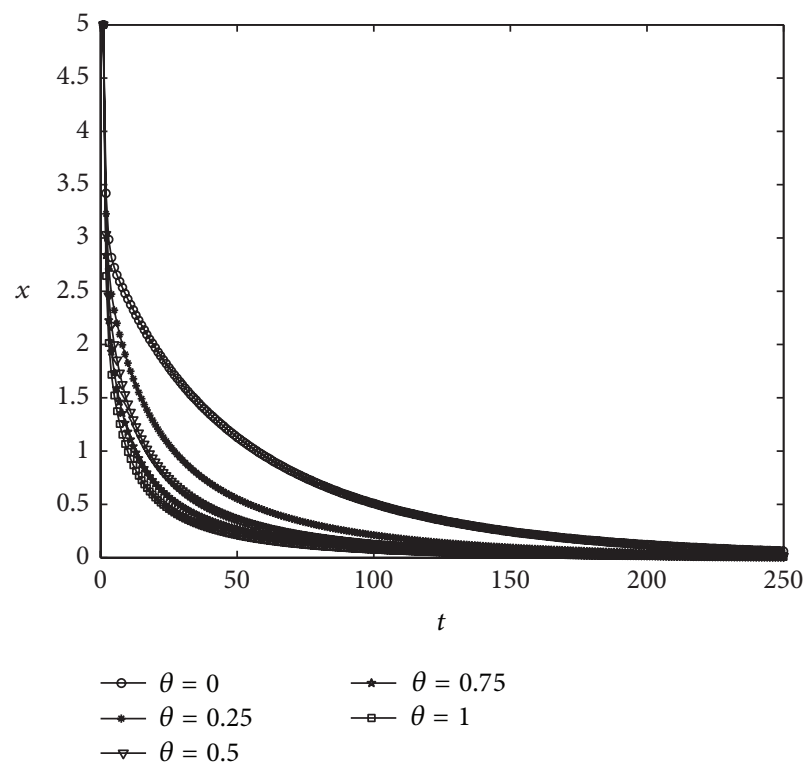

FIGURE 1: Illustration of the effects of pesticide application timing on the extinction of the host population. The parameter values are as follows: $T=10, T_{h}=0.5, K=50, \delta=0.3, q=0.94, \gamma=1, \alpha=0.007$, and $r=2.8$.

Furthermore, model (4) may have a boundary equilibrium $\left(x^{*}, 0\right)$, that is, parasitoid-free equilibrium, if $x^{*}$ satisfies the following equation:

$$
(1-q)\left[\theta f\left(x^{*}\right)+(1-\theta) f\left((1-q) x^{*}\right)\right]=1
$$

that is, we have

$$
\begin{aligned}
& (1-q)\left[\theta \exp \left(r\left(1-\frac{x^{*}}{K}\right)\right)\right. \\
& \left.\quad+(1-\theta) \exp \left(r\left(1-\frac{(1-q) x^{*}}{K}\right)\right)\right]=1 .
\end{aligned}
$$

Note that it is not easy to find the sufficient conditions of the existence of $x^{*}$ for the above equation, so we first consider the following two special cases: (a) $\theta=0$ and (b) $\theta=1$. The analytical formula for $x^{*}$ can be easily obtained as follows:

(i) $x_{1}^{*}=(K / r)(r-\ln (1 /(1-q)))$ for $\theta=1$.

(ii) $x_{0}^{*}=(K / r(1-q))(r-\ln (1 /(1-q)))$ for $\theta=0$.

Note that, for the existence of $x^{*}$ in the above two special cases, we need

$$
r-\ln \left(\frac{1}{1-q}\right)>0
$$

that is, $x^{*}$ is positive provided $(1-q) e^{r}>1$, which means that $x^{*}$ exists if $(0,0)$ is unstable in those special cases. Obviously, we have $x_{0}^{*}>x_{1}^{*}$.

It follows from

$$
\begin{aligned}
& (1-q)\left[\theta \exp \left(r\left(1-\frac{x^{*}}{K}\right)\right)\right. \\
& \left.+(1-\theta) \exp \left(r\left(1-\frac{(1-q) x^{*}}{K}\right)\right)\right]=1
\end{aligned}
$$

that

$$
\begin{aligned}
& e^{r}(1-q) \\
& \cdot\left[\theta \exp \left(-\frac{r x^{*}}{K}\right)+(1-\theta) \exp \left(-\frac{r(1-q) x^{*}}{K}\right)\right]
\end{aligned}
$$$$
=1 \text {, }
$$

from which we can see that the necessary condition for existence of $x_{\theta}^{*}$ is $e^{r}(1-q)>1$.

For stability of the boundary equilibrium $\left(x^{*}, 0\right)$, the two eigenvalues of Jacobian matrix are as follows:

$$
\begin{aligned}
\lambda_{1} & =(1-q)\left(\theta \exp r\left(1-\frac{x^{*}}{K}\right)\right. \\
& \left.+(1-\theta) \exp r\left(1-\frac{(1-q) x^{*}}{K}\right)\right) \\
& -\frac{(1-q) x^{*}}{K}\left(\theta r \exp r\left(1-\frac{x^{*}}{K}\right)\right. \\
& \left.+(1-\theta) r(1-q) \exp r\left(1-\frac{(1-q) x^{*}}{K}\right)\right)=1 \\
& -\frac{(1-q) x^{*}}{K}\left(\theta r \exp r\left(1-\frac{x^{*}}{K}\right)\right. \\
& \left.+(1-\theta) r(1-q) \exp r\left(1-\frac{(1-q) x^{*}}{K}\right)\right), \\
\lambda_{2} & =\frac{(1-q) a T x^{*}}{1+a T_{h}(\theta+(1-\theta)(1-q)) x^{*}}+\delta .
\end{aligned}
$$

Obviously, we have $\lambda_{1}<1$ and $\lambda_{2}>0$. 


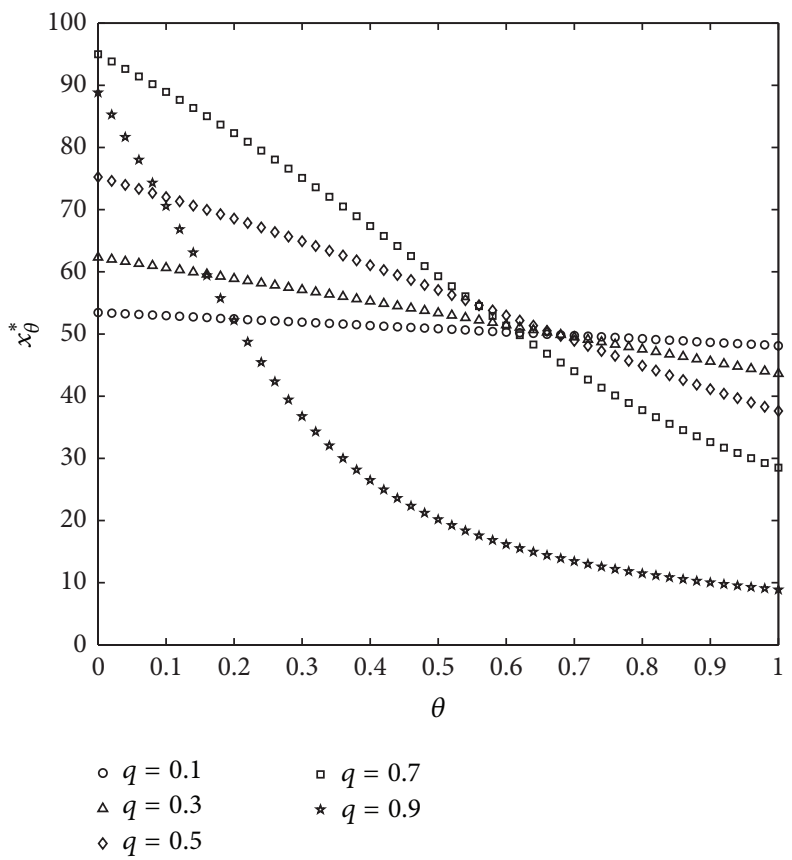

FIgURE 2: The effects of instant killing rate $q$ and pesticide application time $\theta$ on the values of $x_{\theta}^{*}$. The parameter values are as follows: $T=10, T_{h}=0.5, K=50, \delta=0.3, q=0.1, \gamma=1, \alpha=0.007$, and $r=2.8$.

What we are interested in is how the parameters including $\theta$ and $q$ affect the values of $x^{*}$ and its stability. To address this, we fix the parameter values as those in Figure 2 and plot $x^{*}$ against the parameter $\theta$ for given $q$ (denoted by $x_{\theta}^{*}$ ). It follows from Figure 2 that the values of $x^{*}$ are significantly changed as $\theta$ varies for large $q$ (as shown in Figure 2 for $q=0.9$ ), and $x_{\theta}^{*}$ is a monotonically decreasing function of $\theta$. All those indicate that carefully selecting the pesticides and designing the application time are quite important in pest control.

Therefore, we consider $x^{*}$ as the function of both parameters $\theta$ and $q$ (denoted by $x_{q, \theta}^{*}$ ) and fix all other parameters as those in Figure 3(a). Meanwhile, the two eigenvalues $\lambda_{1}$ and $\lambda_{2}$ are also plotted in Figure 3(b). It follows from Figure 3 that the values of $x_{q, \theta}^{*}$ and their stabilities can be strongly influenced by the instant killing rate $q$ and pesticide application time $\theta$. Note that beyond the stable regions of $x_{q, \theta}^{*}$ the dynamical behavior of model (4) could be very complex. Therefore, we will address the complexity of dynamics by employing the bifurcation analysis in the following.

\section{Bifurcation Analysis and Complex Dynamics}

It follows from [19] that the rich dynamic behavior for model (4) can have multiple attractors coexistence and complex bifurcations. However, what we are interested in is how the key parameters of model (4) affect the successful pest control and biological implications. In particular, we will focus on the effects of the intrinsic growth rate, instant killing rate, timing of pesticide applications, and the initial densities of host and parasitoid populations on successful biological control in this section by carrying bifurcation analyses.
Firstly, we show the bifurcation diagrams with respect to the intrinsic growth rate for different instant killing rate in Figure 4. Figure 4(a) is similar to the bifurcation diagram shown in [19], from which we can see that model (4) has quite complex dynamics including chaotic windows, periodic windows, and quasi-periodic windows as parameter $r$ varies. Moreover, the different attractors can coexist for certain range of parameter values of $r$. When we slightly increase parameter $q$ from 0 to 0.05 , we find that the bifurcation diagram is significantly changed. In particular, the multiple attractors of the host and parasitoid populations can coexist with a wide range of parameter $r$, as shown in Figure 4(b).

To show the effects of changing the parameter values on different attractors in more detail, we plot different attractors in Figure 5, from which we can see that the instant killing rate can significantly influence the final state of host-parasitoid populations, as shown in Figures 5(a) and 5(b). Similarly, the intrinsic growth rate can also affect the final state of hostparasitoid populations, as shown in Figures 5(c) and 5(d).

Thus, it follows from Figure 4 that different hostparasitoid initial densities or host-parasitoid ratios can significantly affect the dynamical behavior of systems (4). For example, the solutions of model $(4)$ starting from $(5,5)$ and $(2,1)$ will tend to two different attractors for fixed parameter values, as shown in Figure 6. Similar results can be obtained for the fixed parameter values in Figure 7. Those further clarify that the initial values play a key role in determining the final states of host-parasitoid populations.

In order to address in more detail how the initial values affect the final states of host and parasitoid populations, we provide the basins of attraction of two attractors shown in Figures 6 and 7, respectively, for a quite wide range of 


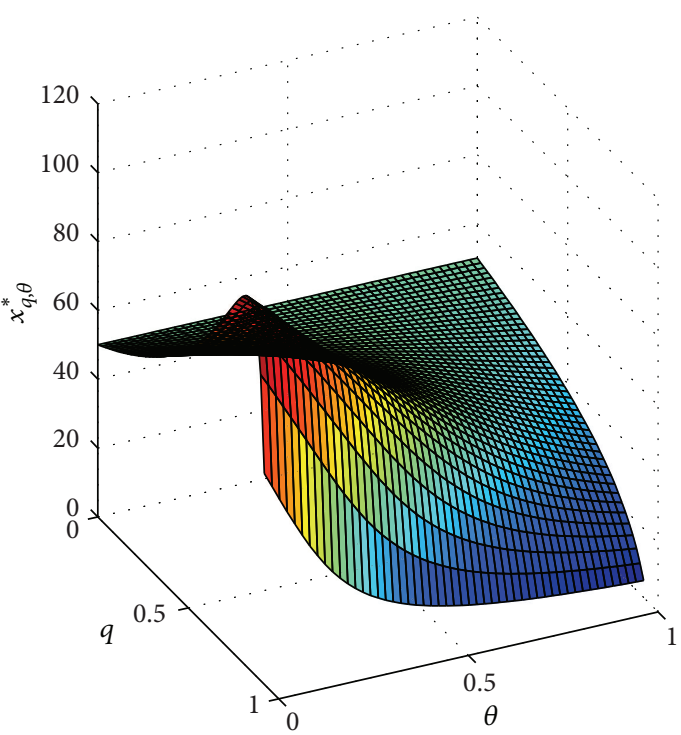

(a)

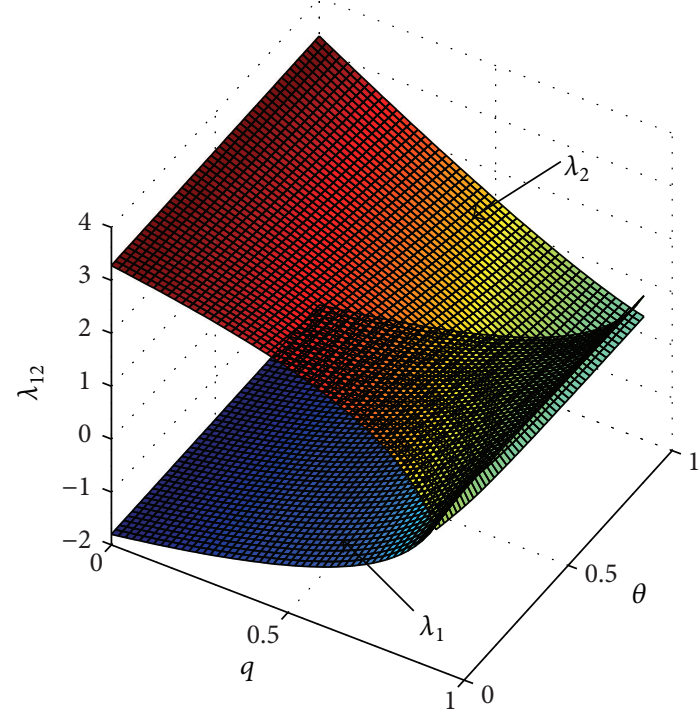

(b)

FIGURE 3: The effects of instant killing rate $q$ and pesticide application time $\theta$ on the values of $x_{q, \theta}^{*}$ and its stability. The parameter values are as follows: $T=10, T_{h}=0.5, K=50, \delta=0.3, q=0.1, \gamma=1, \alpha=0.007$, and $r=2.8$.

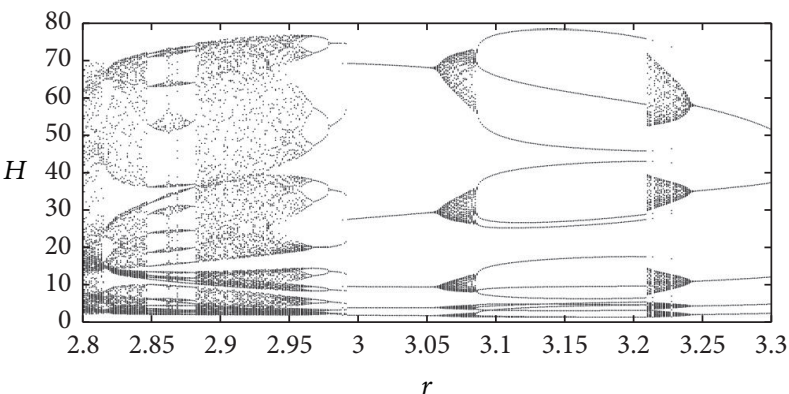

(a)

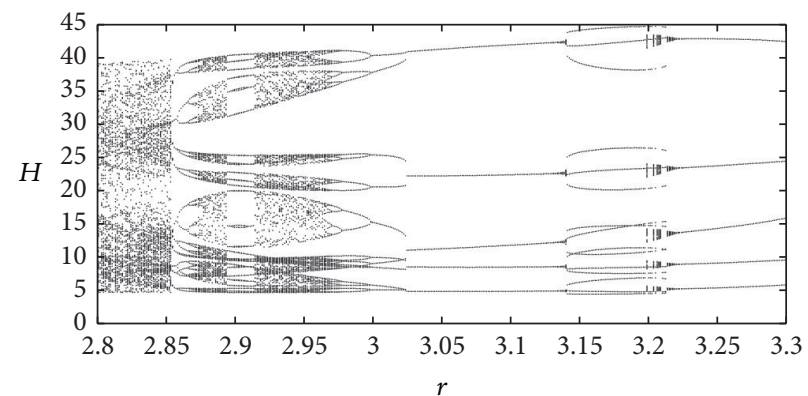

(b)

FIGURE 4: Bifurcation diagrams with respect to the intrinsic growth rate $r$ with different $q$ values. The parameter values are as follows: $T=$ $10, T_{h}=0.5, K=50, \delta=0.3, \gamma=1, \alpha=0.01, \theta=0, q=0$ in (a), and $q=0.05$ in (b).

initial values of host and parasitoid population. The basins of attraction shown in Figures 8 and 9 indicate that the patterns of basins are quite complex and the final states of both host and parasitoid population can be influenced by slightly changing the initial values, which could result in complexity during the pest control due to random perturbation.

The bifurcation diagrams with respect to survival rate $\delta$ given in Figure 10 show that the survival rate of the parasitoid population can greatly affect the dynamic behavior of model (4). Moreover, the periodicity could be strengthened as parameter $\delta$ increases and complexity can be reduced, which means that the larger the survival rate, the stronger the stability of model (4).

Furthermore, the bifurcation diagram with respect to the searching rate $\alpha$ shows that model (4) exists as a boundary equilibrium for small $\alpha$ (i.e., parasitoid-free equilibrium), and then model (4) has an interior equilibrium as $\alpha$ increases and exceeds some critical values (as shown in Figure 11). In this case, both host and parasitoid populations can coexist and stabilize at the interior equilibrium. The quasi-periodic solution appears once we further increase the parameter $\alpha$, which could exist for a wide region of parameters.

\section{Sensitivity Analysis and Key Parameters}

In this section, what we would like to address is how to determine the key parameters which can significantly affect the dynamics and consequently affect the pest control. Therefore, we employ the uncertainty and sensitivity analyses methods in this section. We first consider the simple case before we do this. 


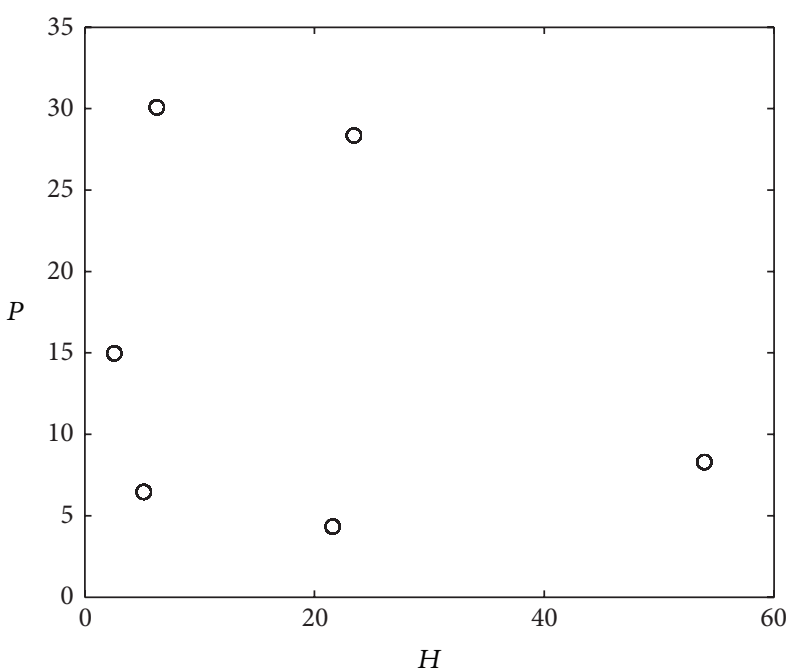

(a) $q=0$

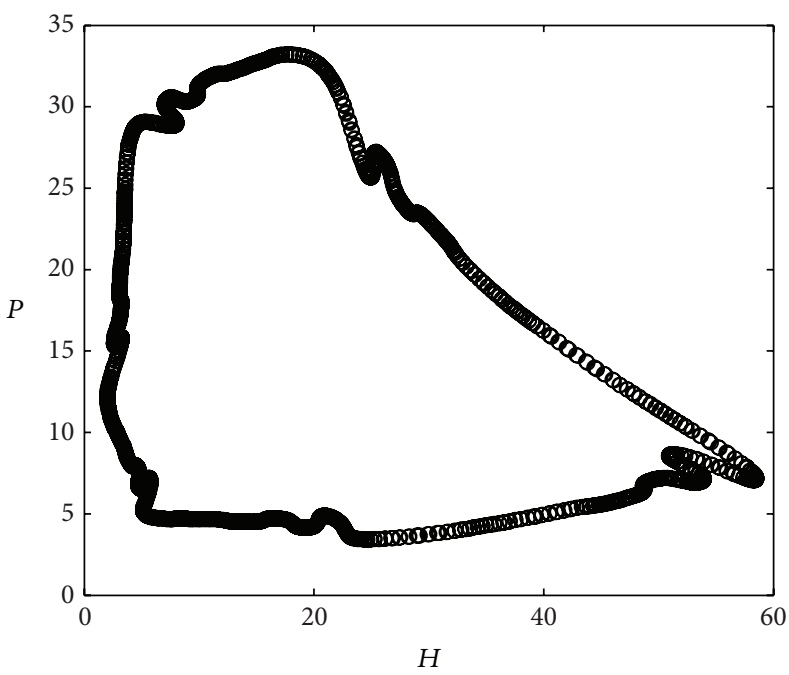

(c) $q=0.05$

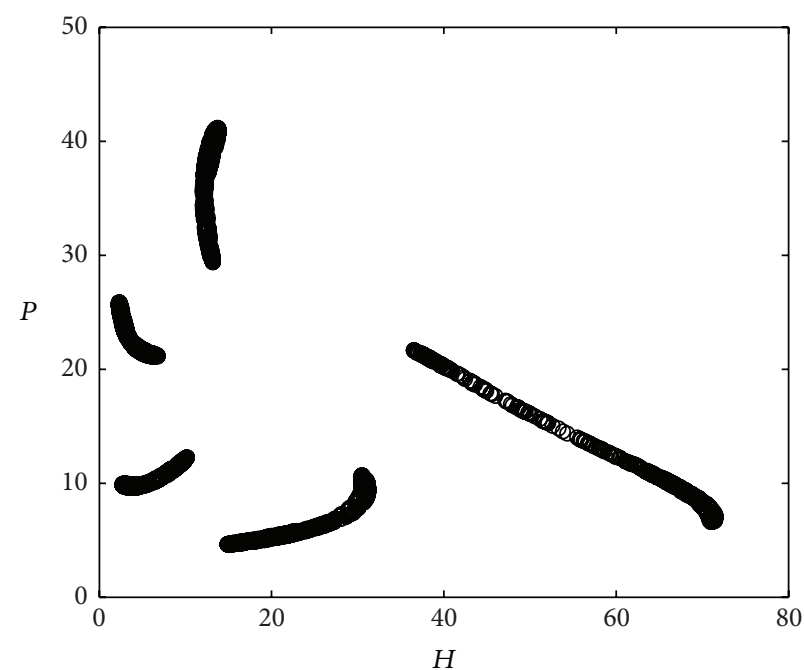

(b) $q=0$

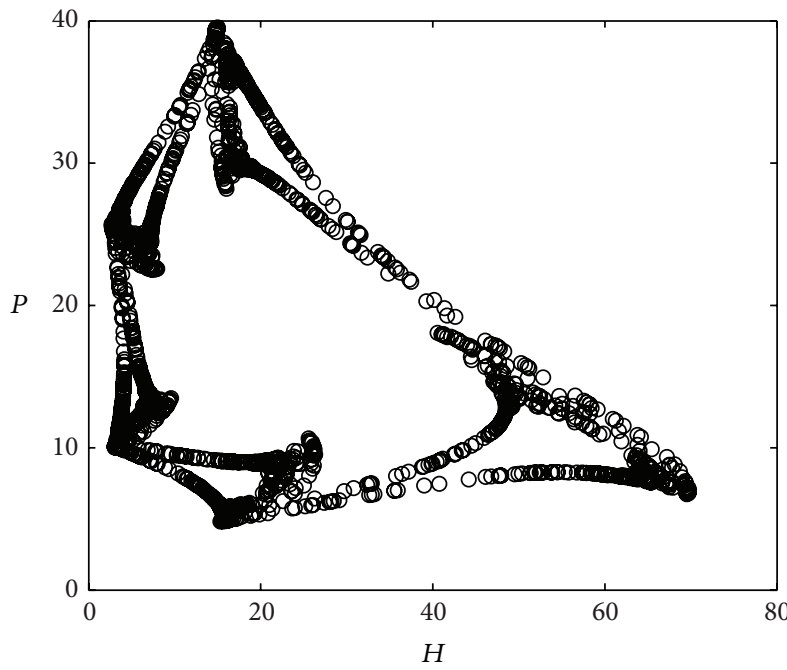

(d) $q=0.05$

Figure 5: Attractors of model (4) for different parameter values $r$ and $q$. The parameter values are as follows: $T=10, T_{h}=0.5, K=50, \delta=$ $0.3, \gamma=1, \alpha=0.01, \theta=0$, and $q=0$ in (a-b), $q=0.05$ in (c-d), $r=2.3$ in (a, c), and $r=2.84$ in (b, d).

The results shown in Figure 12 based on the baseline parameter values in Figure 11 indicate that slightly changing the parameter values $\alpha$ and $\theta$ can strongly influence the stability of both host and parasitoid populations. Those can be confirmed by comparing Figures 12(a) and 12(b) with Figures $12(\mathrm{c})$ and $12(\mathrm{~d})$, from which we can see that the slight increase of the value of $\alpha$ can result in strong oscillation of solutions for model (4).

To address those in more detail, in the following the sensitivity analysis was performed by evaluating the PRCCs $[27,28]$ for various input parameters against the solutions of both the host and parasitoid populations where we have calculated the values at 1000th generations, and then the most significant parameters (such as killing efficiency rate $q$ and pesticide application time $\theta$ ) were determined. A type of stratified Monte Carlo sampling method, named as LHS method, first proposed by Marino et al. [28] has been employed here, which has been applied to deterministic mathematical models [27]. PRCC can measure the influence of uncertainty in estimating the values of the input parameter on the imprecision in predicting the value of the output variable $[27,28]$. Therefore, we performed uncertainty and sensitivity analyses for all parameters in model (4) using LHS with 1000 samples. A norm distribution function was used and tested for significant PRCCs for all parameters with mean values (i.e., the baseline values) as shown in Figures 13 and 14.

PRCC results and PRCC scatter plots of all the parameters involving in model (4) have been provided in Figures 13 and 14 for both the host and parasitoid populations, respectively. In both figures the first row shows the PRCC 


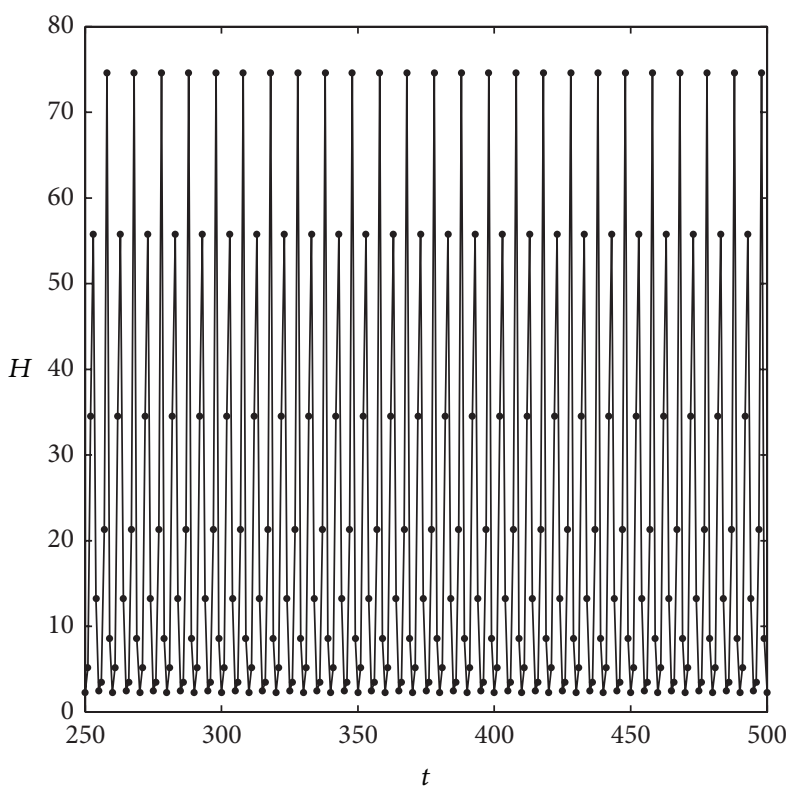

(a)

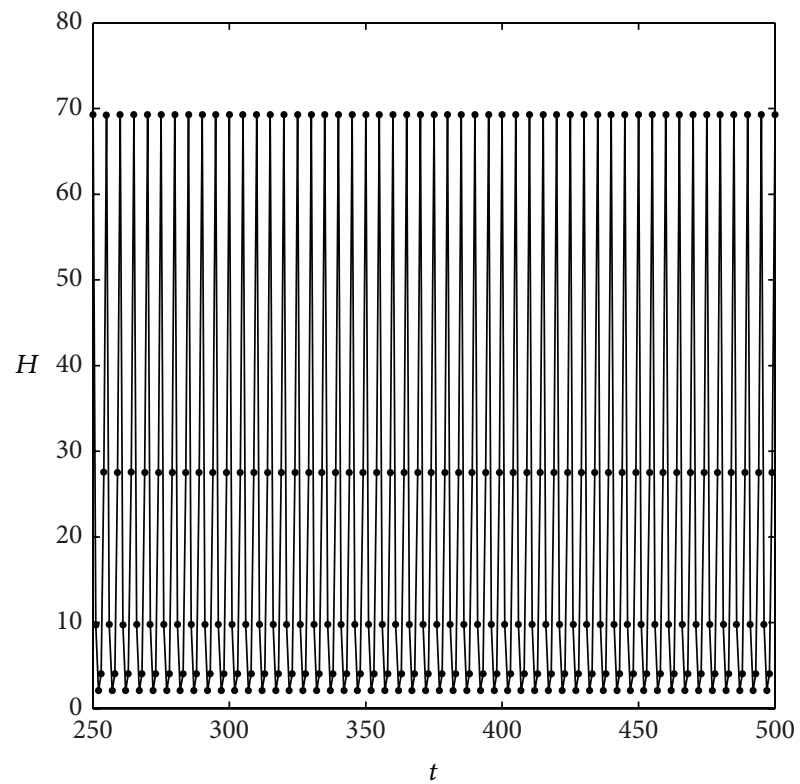

(c)

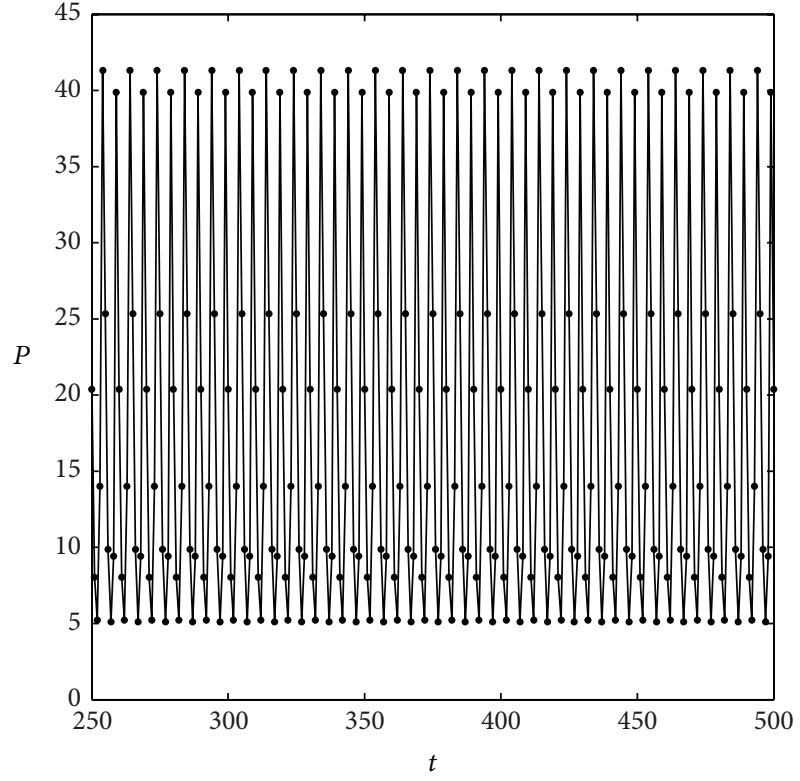

(b)

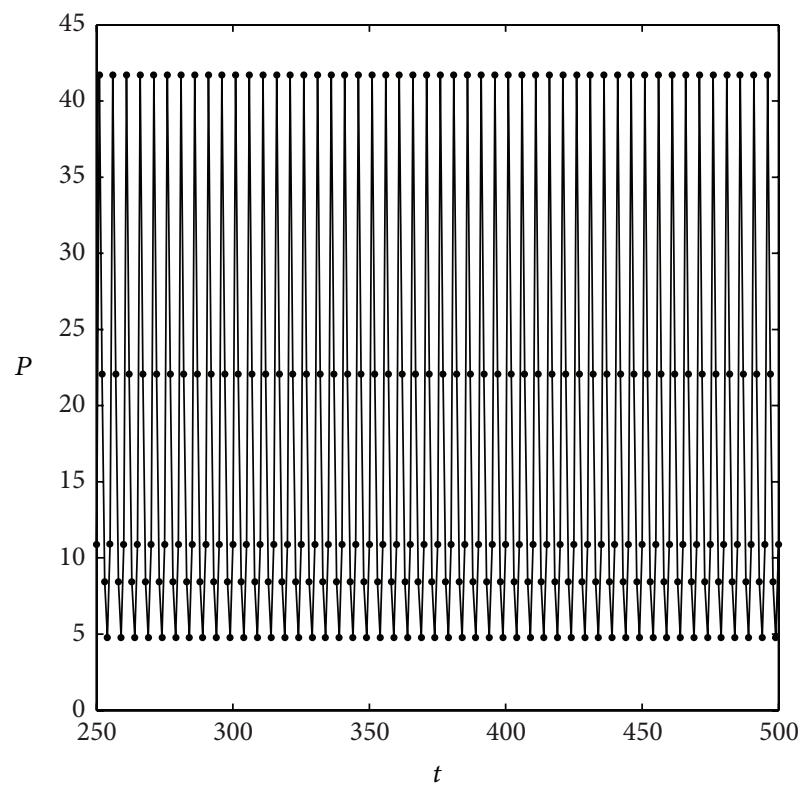

(d)

Figure 6: Two attractors' coexistence. The parameter values are as follows: $T=10, T_{h}=0.5, K=50, \delta=0.3, \gamma=1, \alpha=0.01, \theta=0, q=0$, and $r=2.99$. The initial values are $(5,5)$ for $(a-b)$ and $(1,2)$ for $(c-d)$.

results with sample size 1000 and all parameters were varied simultaneously. The second and third rows provide the PRCC scatter plots with sampling size 1000 and significant $p<$ 0.01 . We considered absolute values of PRCC greater than 0.4 as indicating an important correlation between input parameters and output variables, values between 0.2 and 0.4 as moderate correlations, and values between 0 and 0.2 as not significantly different from zero. The positive sign of their PRCCs indicates that if the parameters are increased, the value of the solution at that generation increases (and vice versa). The negative sign suggests that if increased, the value of the solution at that generation decreases (and vice versa). All those show that the signs of PRCC values related to all parameters can help us to design the pest control strategies based on the sensitivity of each parameter with respect to the solution of model (4).

It follows from Figures 13 and 14 that the parameters $K$, $r$, and $q$ are responsible for increasing the values of the host solution at 1000 th generation. The PRCC value for killing rate $q$ is a paradox result for pest control [23], because the larger 


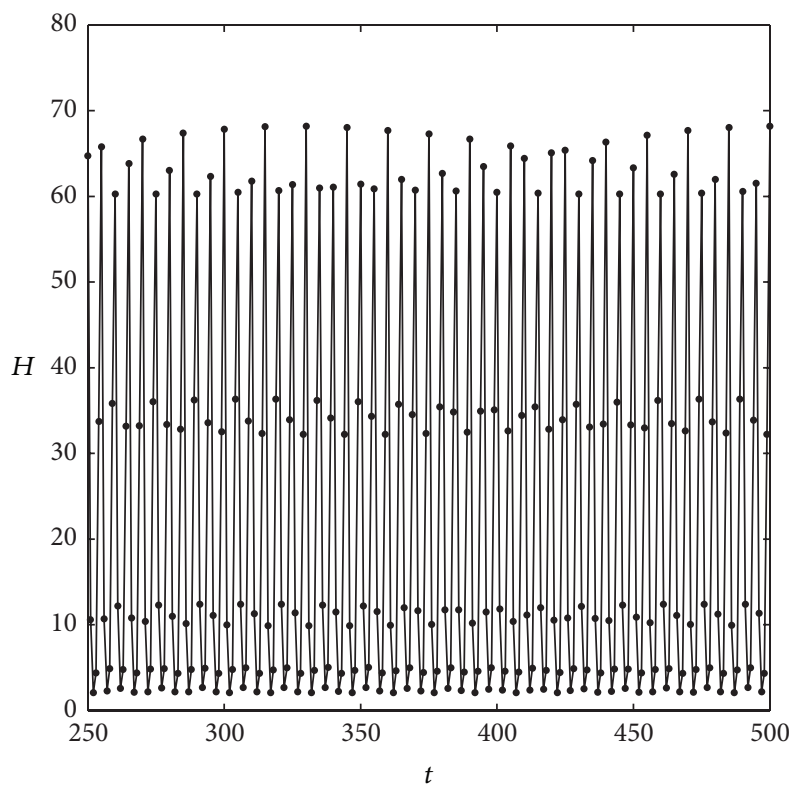

(a)

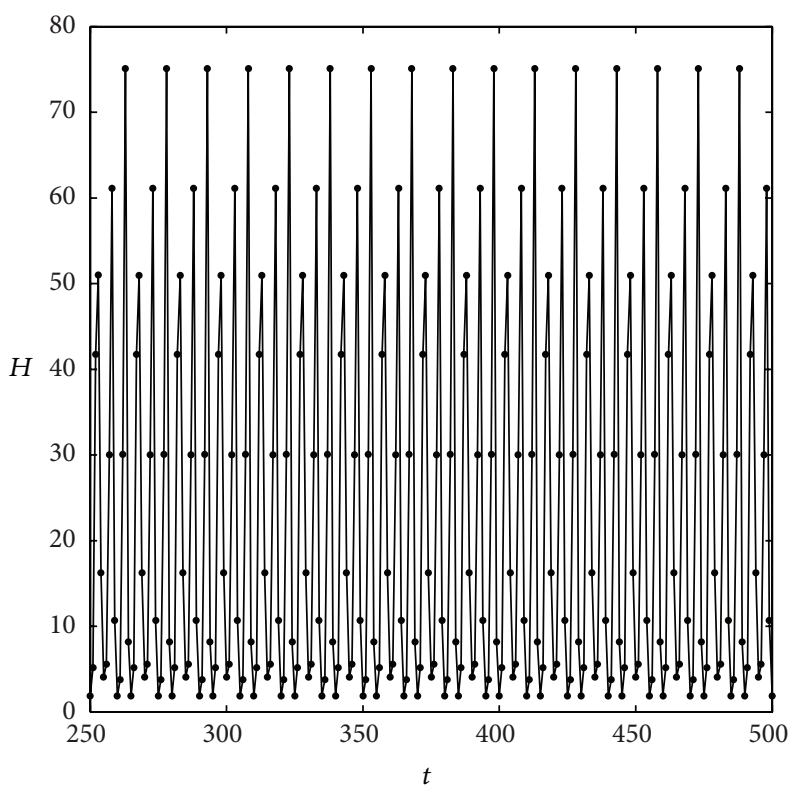

(c)

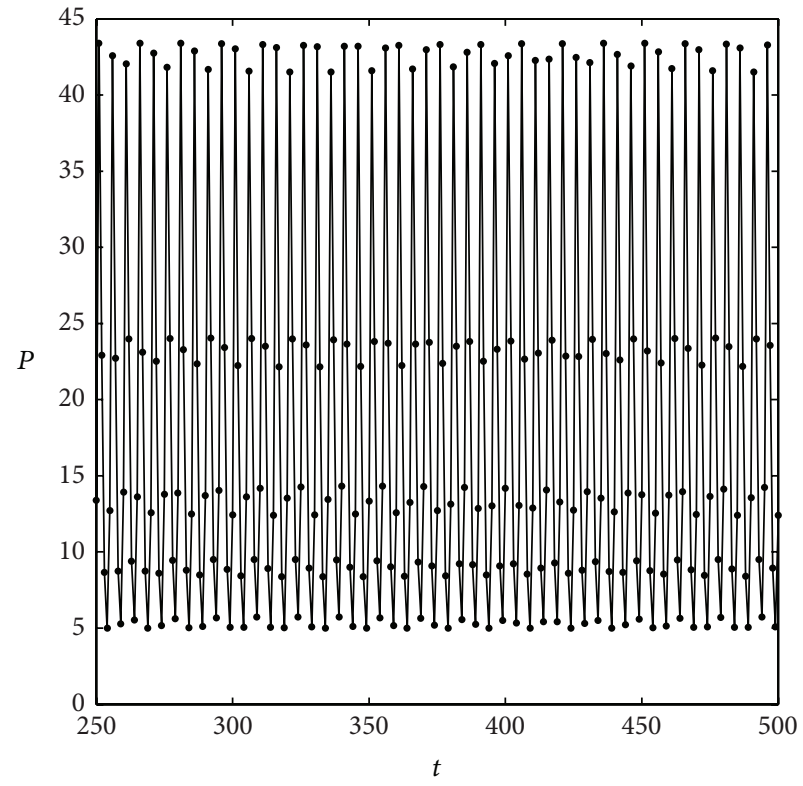

(b)

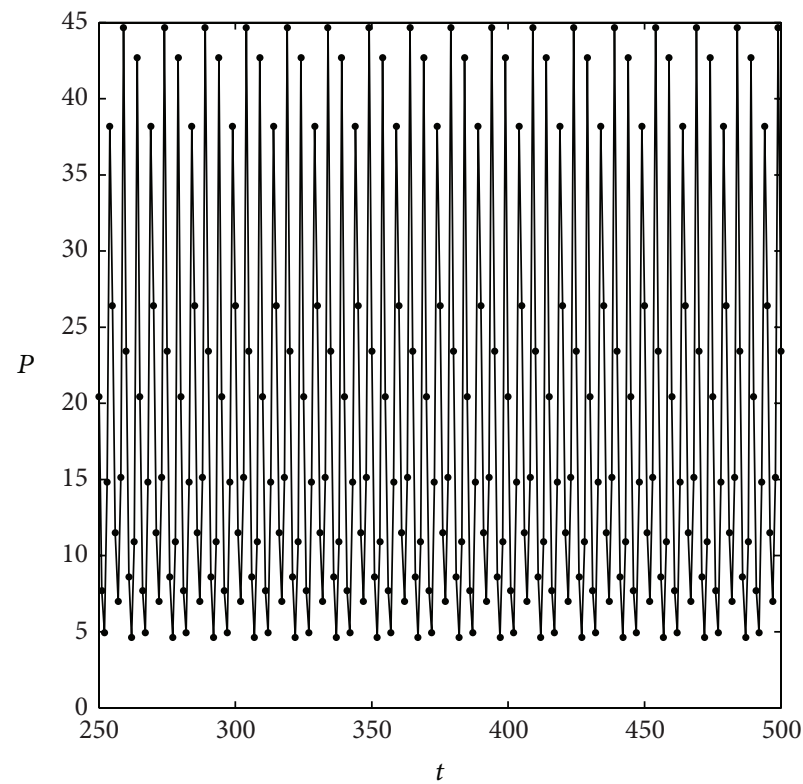

(d)

Figure 7: Two attractors' coexistence. The parameter values are as follows: $T=10, T_{h}=0.5, K=50, \delta=0.3, \gamma=1, \alpha=0.01, \theta=0, q=$ 0.05 , and $r=3.2$. The initial values are $(5,5)$ for $(a-b)$ and $(2,1)$ for $(c-d)$.

$q$ value is, the larger host population size we have. The main reason is that we apply the chemical control strategy at the end of each season or generation; that is, $\theta=1$ or close to one. The parameters $T, \alpha$, and $\delta$ are responsible for decreasing the values of the host solution at 1000th generation, so increasing all those parameters are beneficial for pest control. The most significant control parameters for the host population are $T$, $K, \alpha$, and $\delta$ (see Figure 13).

The sign of PRCC value of the parameter $q$ related to the parasitoid population is negative and is important correlation to the size of the parasitoid population, which shows that increasing the killing rate for the host population may result in reducing the number of parasitoid populations, and in turn the host could outbreak again. The effects of all the other parameters on the parasitoid population have been provided in Figure 14.

\section{Biological Conclusions}

In this work we have proposed a discrete host-parasitoid model with pulse chemical control which can be implemented within each pest generation or parasitism season. 


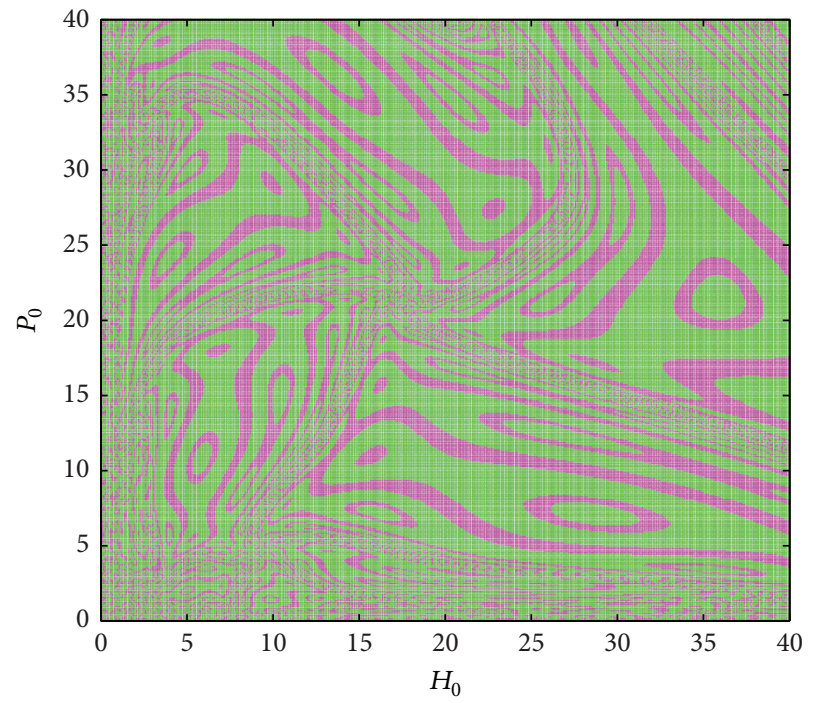

Figure 8: The basins of attraction of model (4) for two attractors shown in Figure 6. The parameter values are as follows: $T=10, T_{h}=$ $0.5, K=50, \delta=0.3, \gamma=1, \alpha=0.01, \theta=0, q=0$, and $r=2.99$.

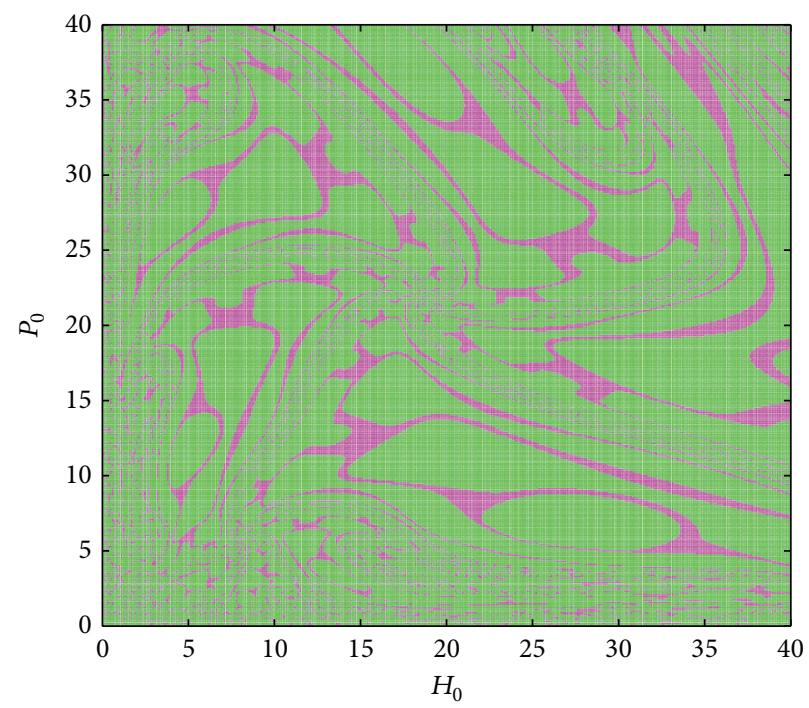

Figure 9: The basins of attraction of model (4) for two attractors shown in Figure 7. The parameter values are as follows: $T=10, T_{h}=$ $0.5, K=50, \delta=0.3, \gamma=1, \alpha=0.01, \theta=0, q=0.05$, and $r=3.2$.

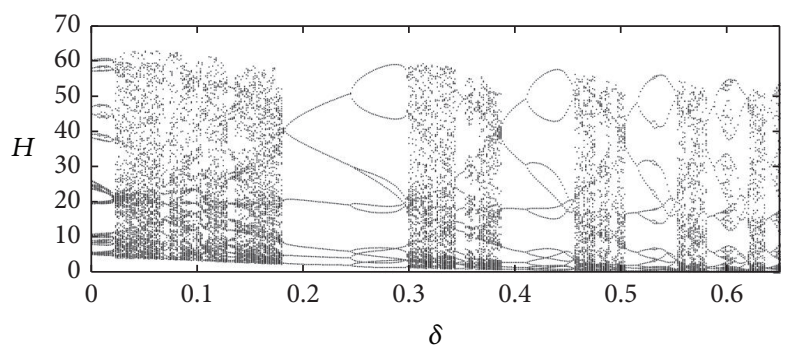

(a)

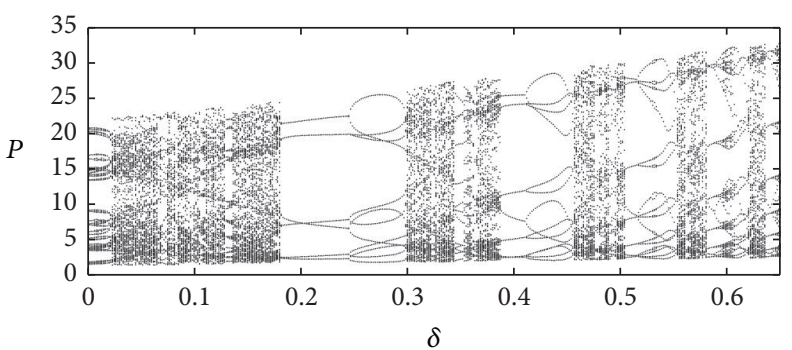

(b)

FIGURE 10: Bifurcation diagram with respect to parameter $\theta$. The parameter values are as follows: $T=10, T_{h}=0.5, K=50, \gamma=1, \alpha=$ $0.015, \theta=1, q=0.2$, and $r=2.6$. 


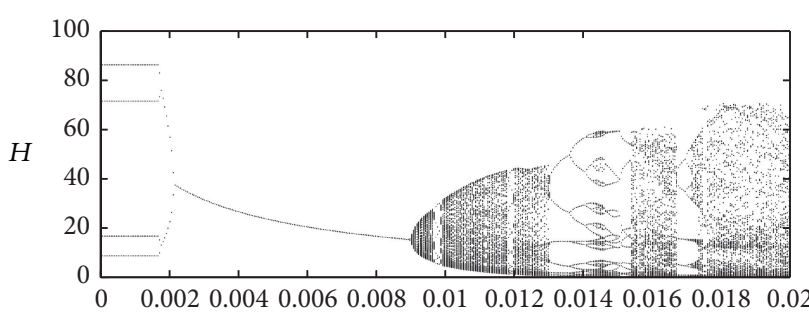

(a)

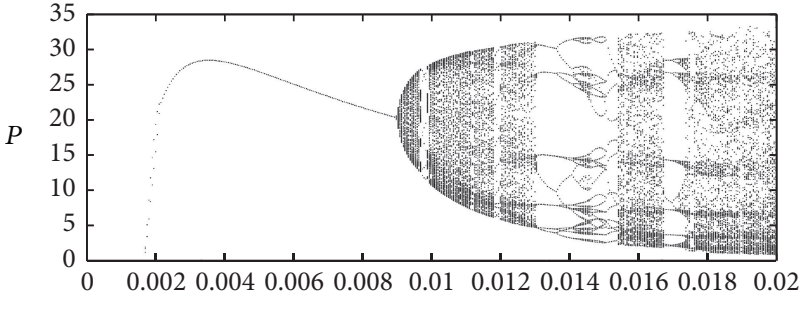

(b)

Figure 11: Bifurcation diagram with respect to parameter $\alpha$. The parameter values are as follows: $T=10, T_{h}=0.5, K=50, \gamma=1, \delta=$ $0.5, \theta=1, q=0.2$, and $r=2.8$.
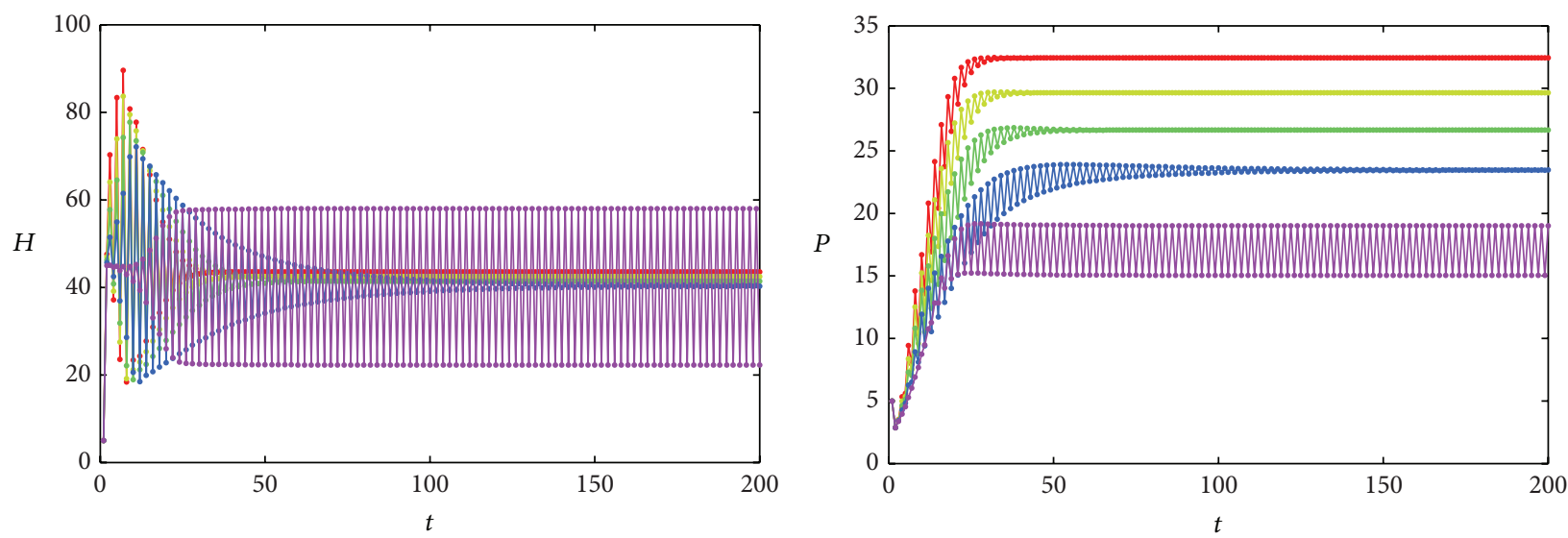

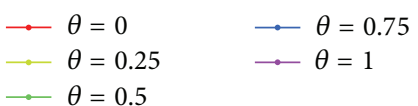

(a) $\alpha=0.002$

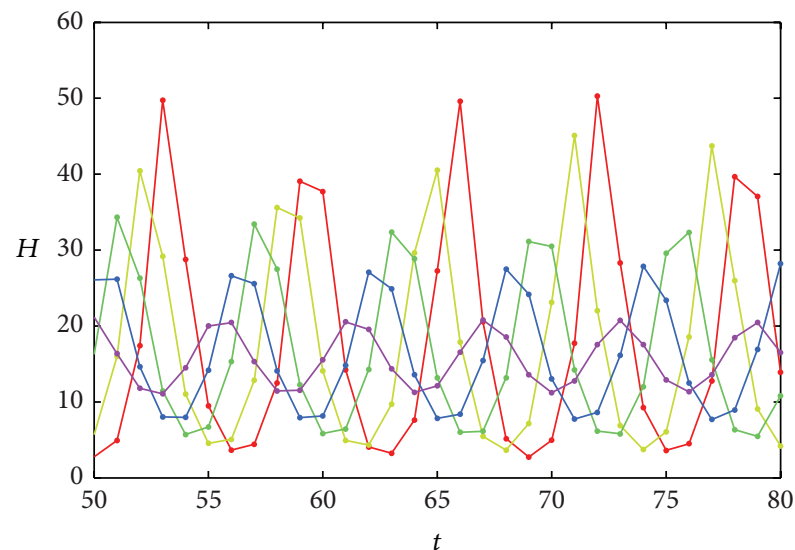

$$
\begin{aligned}
\therefore \theta & =0 \\
\therefore \theta & =0.25 \quad \because \theta=0.75 \\
\because \theta & =0.5
\end{aligned}
$$

(c) $\alpha=0.009$

$$
\begin{aligned}
\because \theta & =0 \\
\because \theta & =0.25 \quad \because \theta=0.75 \\
\because \theta & =0.5
\end{aligned}
$$

(b)

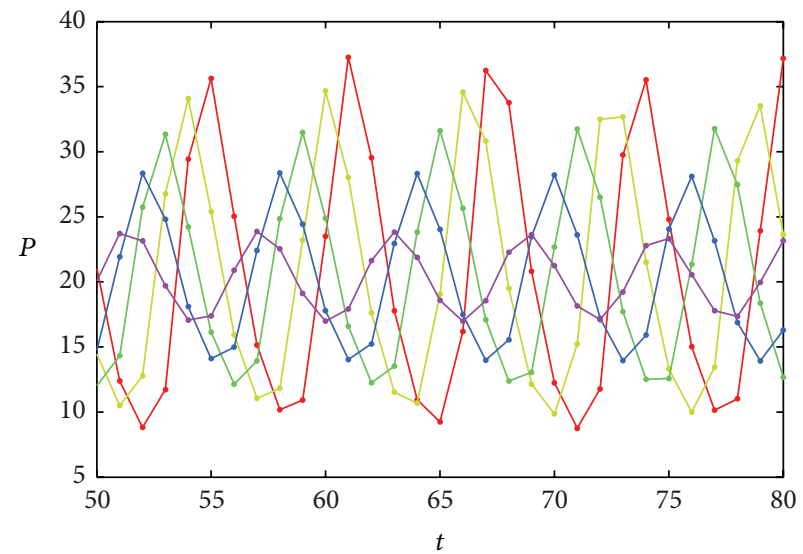

$$
\begin{aligned}
\therefore \theta & =0 \quad \because \theta=0.75 \\
\therefore \theta & =0.25 \quad \because \theta=1 \\
\because \theta & =0.5
\end{aligned}
$$

(d)

FIGURE 12: Sensitivity analyses of both parameter values of $\alpha$ and $\theta$. The parameter values are as follows: $T=10, T_{h}=0.5, K=50, \gamma=1, \delta=$ $0.5, \theta=1, q=0.2$, and $r=2.8$. 

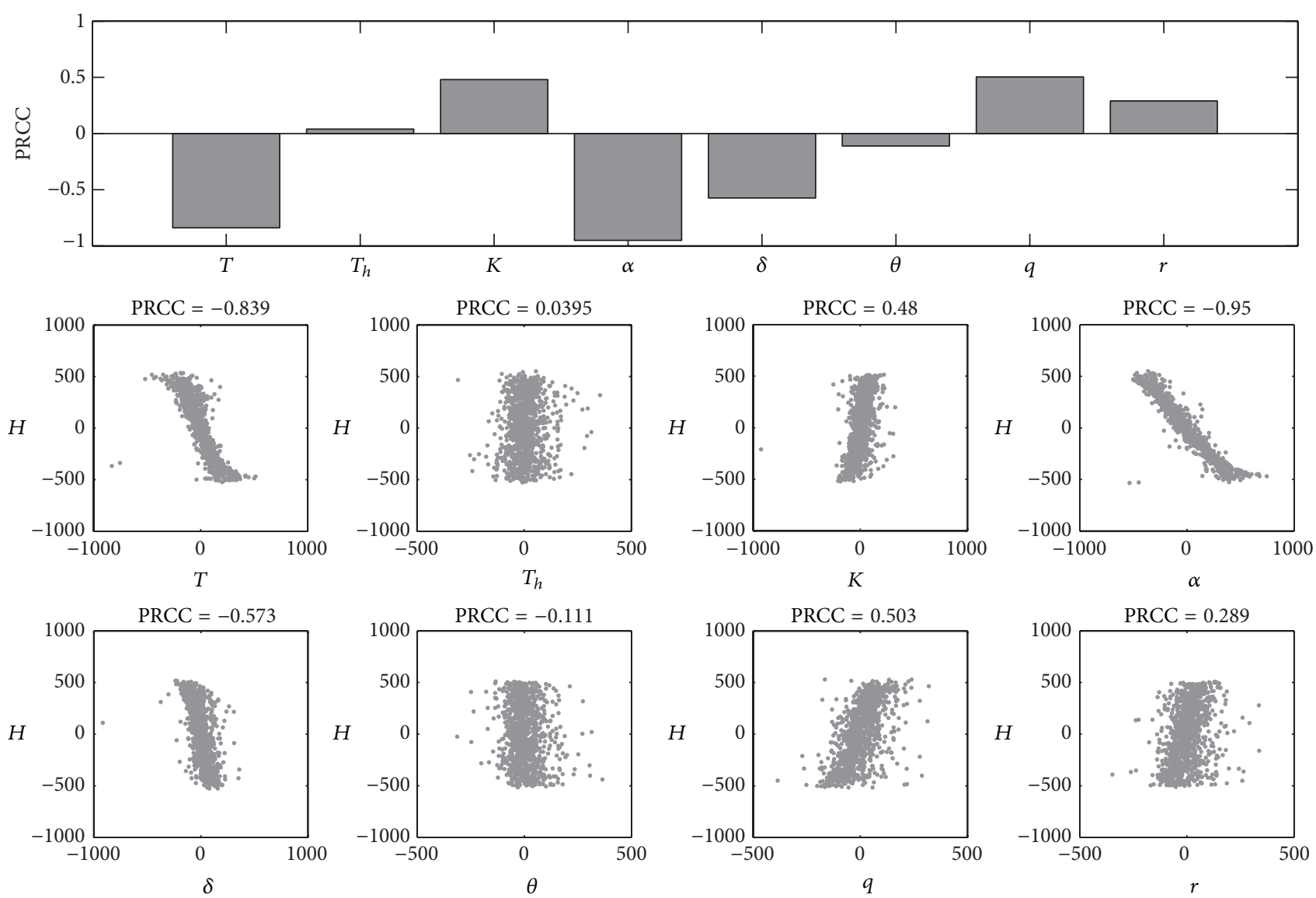

FIGURE 13: Sensitivity analyses based on the PRCC values for the host population at 1000th generation. The baseline parameter values are as follows: $T=10, T_{h}=0.5, K=50, \alpha=0.005, \gamma=1, \delta=0.5, \theta=1, q=0.2$, and $r=2.8$.

This allows us to address the effects of timings of pesticide application and instant killing rate on the successful pest control and on the complex dynamics of proposed model.

Therefore, based on the Holling II host-parasitoid model with a fraction of survival rate of parasitoid from one generation to next, we focus on the effects of timings of pesticide application and killing rate on the host or pest control and on the complex dynamics. To address those, we first discuss the existence and stability of both the host and parasitoid populations extinction equilibrium and parasitoid-free equilibrium, and then the threshold conditions which guarantee the local stabilities have been provided. The threshold condition for the stability of $(0,0)$ equilibrium indicates that it only depends on the intrinsic growth of the host population and the instant killing rate $q$. This shows that both the host and parasitoid populations could be extinct simultaneously if the pesticide is effective enough no matter when we spray the pesticides. But the timing of pesticide applications can significantly influence the asymptotic behavior of solutions of model (4), as shown in Figure 1.

The interesting results shown in Figures 2 and 3 indicate that the different timings of pesticide applications and instant killing rate could result in significant different size of the host population and consequently influences the pest control. All those results confirm the importance of timings of pesticide application when the density dependent is involved into the model. Further, the complex dynamics including multiple attractors coexistence, quasi-periodic windows, chaotic behavior, and initial sensitivity have been studied by using numerical bifurcation analyses. Finally, the uncertainty and sensitivity of all the parameters on the solutions of both the host and parasitoid populations have been investigated, which can help us to determine the key parameters in designing the successful pest control strategy.

This work focused entirely on the host-parasitoid model with chemical control, which means that we only focus on the single control tactic and study its effects on the pest control. Therefore, how can we formulate the host-parasitoid model with IPM strategies? And in particular how can we involve the biological control measure into model (4) with constant releasing rate? All those questions will be considered and studied in the near future, and our findings will be reported elsewhere. 

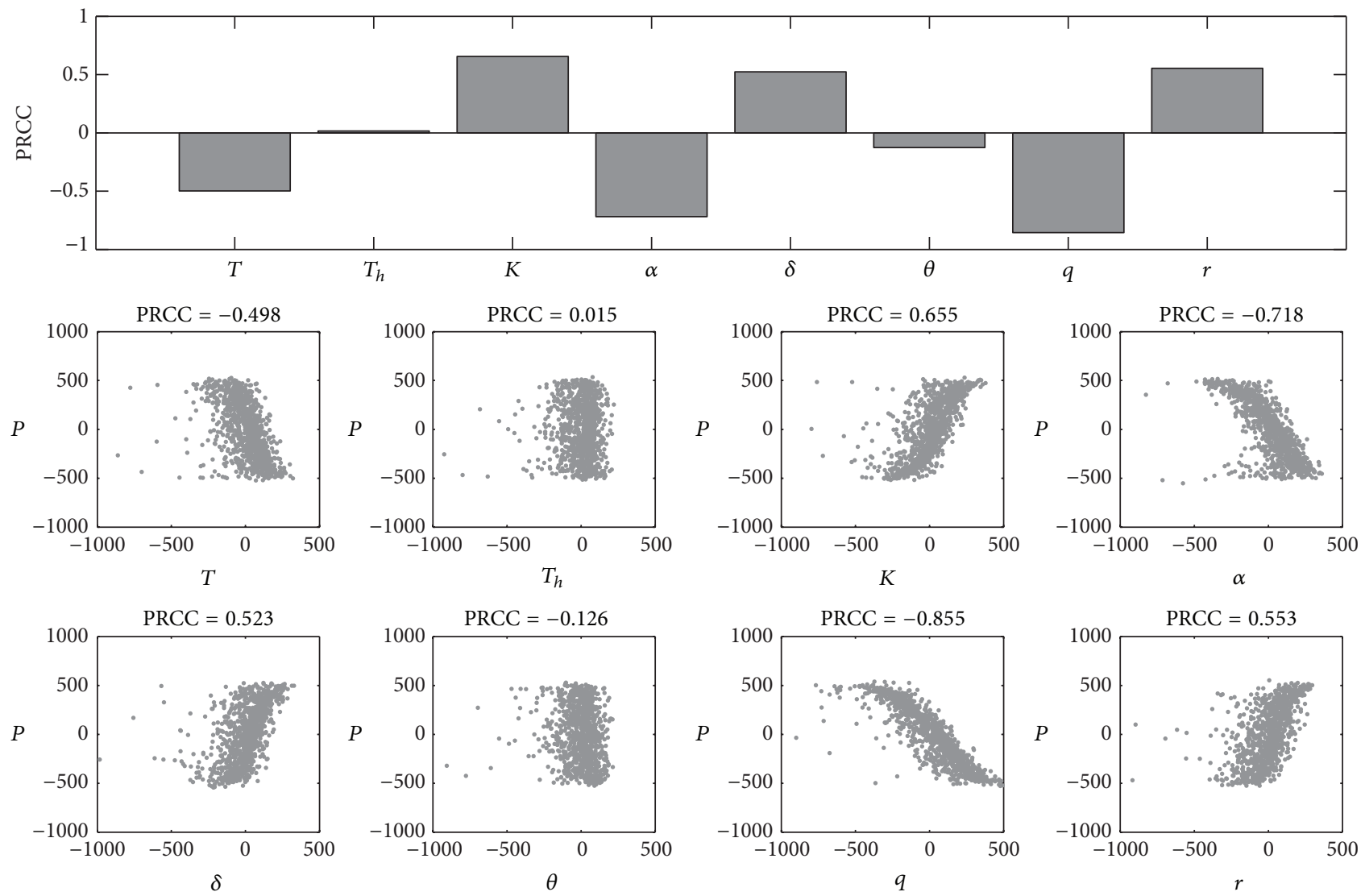

FIGURE 14: Sensitivity analyses based on the PRCC values for the parasitoid population at 1000th generation. The baseline parameter values are as follows: $T=10, T_{h}=0.5, K=50, \alpha=0.005, \gamma=1, \delta=0.5, \theta=1, q=0.2$, and $r=2.8$.

\section{Competing Interests}

The authors declare that they have no competing interests.

\section{References}

[1] S. Y. Tang, Y. N. Xiao, L. S. Chen, and R. A. Cheke, "Integrated pest management models and their dynamical behaviour," Bulletin of Mathematical Biology, vol. 67, no. 1, pp. 115-135, 2005.

[2] J. C. Van Lenteren, "Integrated pest management in protected crops," in Integrated Pest Management, D. Dent, Ed., pp. 311-320, Chapman \& Hall, London, UK, 1995.

[3] J. C. Van Lenteren, "Measures of success in biological control of arthropods by augmentation of natural enemies," in Measures of Success in Biological Control, S. Wratten and G. Gurr, Eds., pp. 77-89, Kluwer Academic Publishers, Dordrecht, The Netherlands, 2000.

[4] S. Tang and L. Chen, "Multiple attractors in stage-structured population models with birth pulses," Bulletin of Mathematical Biology, vol. 65, no. 3, pp. 479-495, 2003.

[5] S. Y. Tang and L. S. Chen, "Modelling and analysis of integrated pest management strategy," Discrete and Continuous Dynamical Systems-Series B, vol. 4, no. 3, pp. 759-768, 2004.

[6] S. Y. Tang and R. A. Cheke, "State-dependent impulsive models of integrated pest management (IPM) strategies and their dynamic consequences," Journal of Mathematical Biology, vol. 50, no. 3, pp. 257-292, 2005.
[7] S. Y. Tang, Y. N. Xiao, and R. A. Cheke, "Multiple attractors of host-parasitoid models with integrated pest management strategies: eradication, persistence and outbreak," Theoretical Population Biology, vol. 73, no. 2, pp. 181-197, 2008.

[8] S. Y. Tang and R. A. Cheke, "Models for integrated pest control and their biological implications," Mathematical Biosciences, vol. 215, no. 1, pp. 115-125, 2008.

[9] S. Y. Tang, Y. N. Xiao, and R. A. Cheke, "Effects of predator and prey dispersal on success or failure of biological control," Bulletin of Mathematical Biology, vol. 71, no. 8, pp. 2025-2047, 2009.

[10] S. Y. Tang, G. Y. Tang, and R. A. Cheke, "Optimum timing for integrated pest management: modelling rates of pesticide application and natural enemy releases," Journal of Theoretical Biology, vol. 264, no. 2, pp. 623-638, 2010.

[11] H. J. Barclay, "Models for pest control using predator release, habitat management and pesticide release in combination," Journal of Applied Ecology, vol. 19, no. 2, pp. 337-348, 1982.

[12] J. R. Beddington, C. A. Free, and J. H. Lawton, "Dynamic complexity in predator prey models framed in difference equations," Nature, vol. 255, no. 5503, pp. 58-60, 1975.

[13] J. K. Waage and M. P. Hassell, "Parasitoids as biological control agents-a fundamental approach," Parasitology, vol. 84, no. 4, pp. 241-268, 1982.

[14] J. K. Waage, M. P. Hassell, and H. C. J. Godfray, "The dynamics of pest-parasitoid-insecticide interactions," Journal of Applied Ecology, vol. 22, no. 3, pp. 825-838, 1985. 
[15] K. A. J. White and K. Wilson, "Modelling density-dependent resistance in insect-pathogen interactions," Theoretical Population Biology, vol. 56, no. 2, pp. 163-181, 1999.

[16] M. P. Hassell, "Insecticides in host-parasitoid interactions," Theoretical Population Biology, vol. 26, no. 3, pp. 378-386, 1984.

[17] M. P. Hassell, "Host-parasitoid population dynamics," Journal of Animal Ecology, vol. 69, no. 4, pp. 543-566, 2000.

[18] J. Grasman, O. A. Van Herwaarden, L. Hemerik, and J. C. Van Lenteren, "A two-component model of host-parasitoid interactions: determination of the size of inundative releases of parasitoids in biological pest control," Mathematical Biosciences, vol. 169, no. 2, pp. 207-216, 2001.

[19] S. Y. Tang and L. S. Chen, "Chaos in functional response hostparasitoid ecosystem models," Chaos, Solitons \& Fractals, vol. 13, no. 4, pp. 875-884, 2002.

[20] Y. N. Xiao, D. Z. Cheng, and S. Y. Tang, "Dynamic complexities in predator-prey ecosystem models with age-structure for predator," Chaos, Solitons \& Fractals, vol. 14, no. 9, pp. 1403-1411, 2002.

[21] Y. Xiao and S. Tang, "The effect of initial density and parasitoid intergenerational survival rate on classical biological control," Chaos, Solitons \& Fractals, vol. 37, no. 4, pp. 1048-1058, 2008.

[22] B. Cid, F. M. Hilker, and E. Liz, "Harvest timing and its population dynamic consequences in a discrete single-species model," Mathematical Biosciences, vol. 248, no. 1, pp. 78-87, 2014.

[23] H. Seno, "A paradox in discrete single species population dynamics with harvesting/thinning," Mathematical Biosciences, vol. 214, no. 1-2, pp. 63-69, 2008.

[24] T. Matsuoka and H. Seno, "Ecological balance in the native population dynamics may cause the paradox of pest control with harvesting," Journal of Theoretical Biology, vol. 252, no. 1, pp. 87-97, 2008.

[25] C. S. Holling, "Some characteristics of simple types of predation and parasitism," Canadian Entomologist, vol. 91, no. 7, pp. 385398, 1959.

[26] C. S. Holling, "The functional response of invertebrate predators to prey density," Memoirs of the Entomological Society of Canada, vol. 98, no. 48, pp. 1-86, 1966.

[27] S. M. Blower and H. Dowlatabadi, "Sensitivity and uncertainty analysis of complex models of disease transmission: an HIV model, as an example," International Statistical Review, vol. 62, no. 2, pp. 229-243, 1994.

[28] S. Marino, I. B. Hogue, C. J. Ray, and D. E. Kirschner, "A methodology for performing global uncertainty and sensitivity analysis in systems biology," Journal of Theoretical Biology, vol. 254, no. 1, pp. 178-196, 2008. 


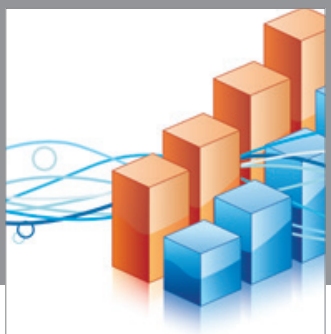

Advances in

Operations Research

vatem alat4

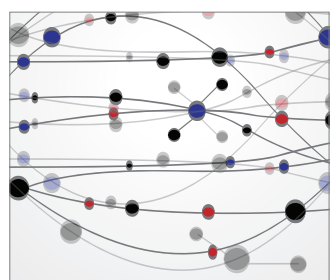

\section{The Scientific} World Journal
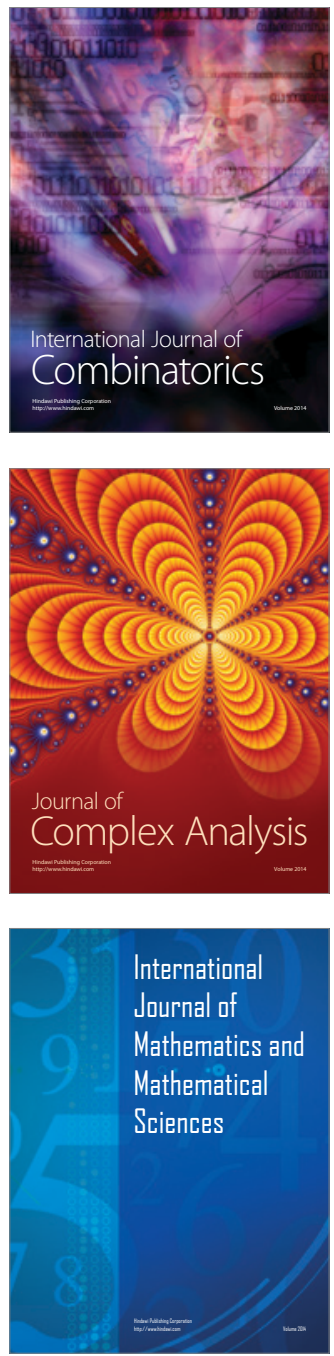
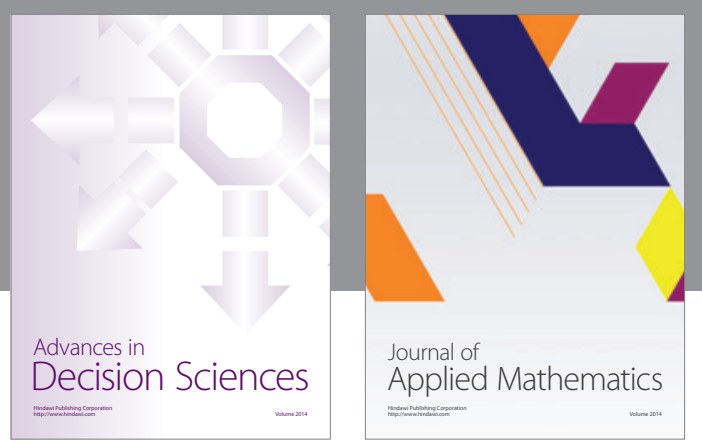

Algebra

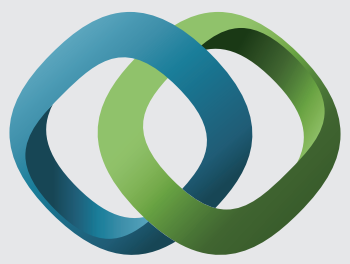

\section{Hindawi}

Submit your manuscripts at

http://www.hindawi.com
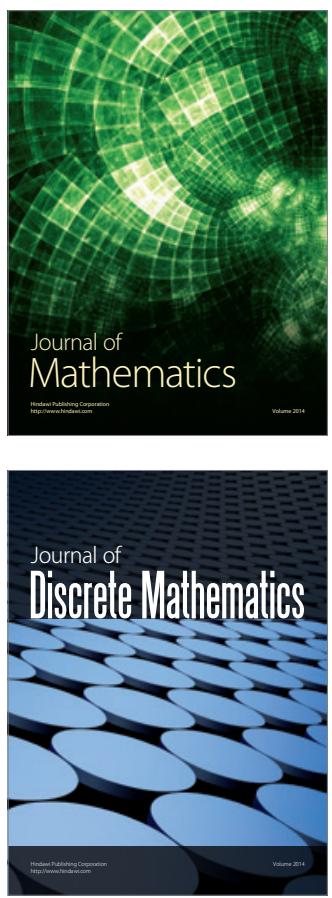

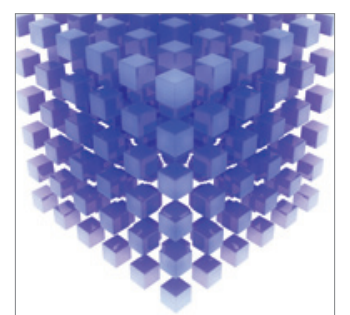

Mathematical Problems in Engineering
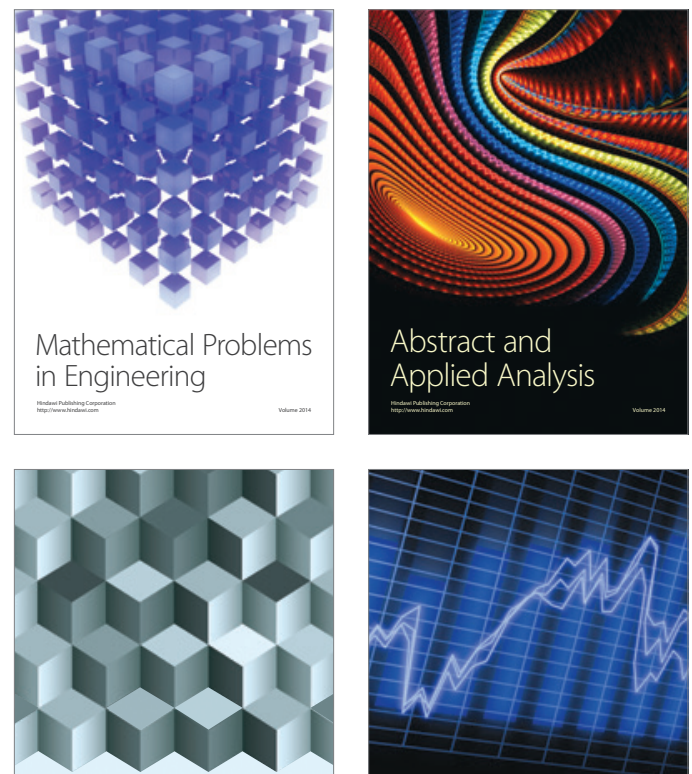

Journal of

Function Spaces

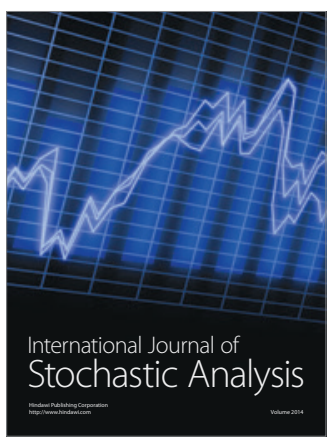

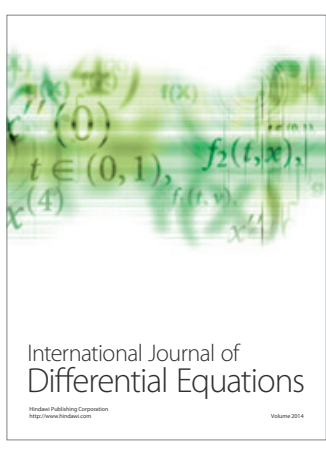
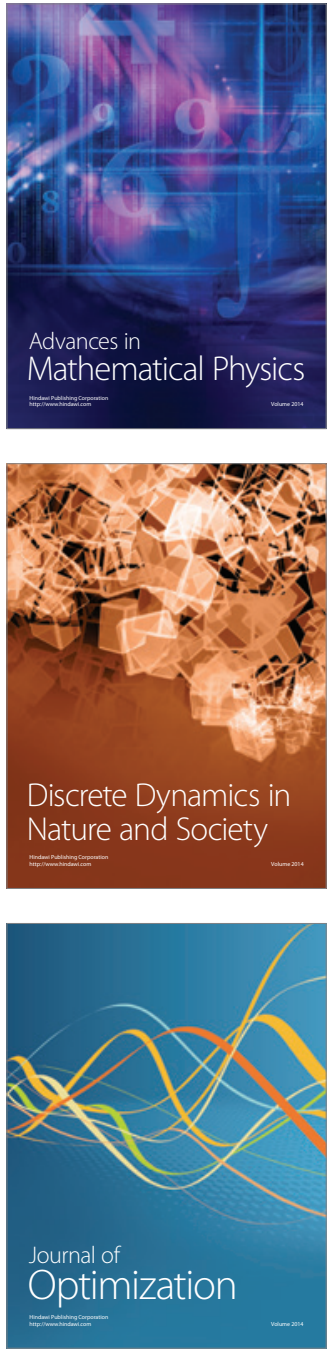